\title{
Post-non-classical science in the age of informatization of society: functional aspect
}

\author{
Liubov Drotianko ${ }^{1, *}$, Mariia Abysova ${ }^{1}$, Nataliia Chenbai $^{1}$ and Tetiana Shorina ${ }^{1}$ \\ ${ }^{1}$ National Aviation University, Kosmonavta Komarova ave., 1, Kyiv, 03058, Ukraine
}

\begin{abstract}
The article researches the transformation of the science functions in the process of changing the scientific rationality type in the conditions of the information society formation. The relevance of the research topic is stipulated by the need for scientific analysis and philosophical understanding of the large-scale changes occurring in modern science under the influence of informatization processes. The theoretical basis of the study are the general philosophical principles - the principles of systematicity, comprehensiveness, concreteness of the study, as well as such socio-philosophical methods as specifically historical, historical-retrospective, comparative-historical method, the method of unity of historical and logical in social cognition. Comparative analysis, interdisciplinary synthesis and other techniques of general scientific methodology are also used. The influence of socio-cultural conditions on the functional transformations of post-non-classical science is substantiated. The basic tendencies of functional transformation of science are revealed: transition to development of complex scientific programs; the convergence of research programs with high-tech manufacturing practices, the integration of computer technologies with methodological tools; the commensuration of subject lines of scientific knowledge with the conditions of formation of socially-human complexes; the coordination of rational-cognitive ethos of science with cultural universals; the extension of the intra-scientific reflection field and the formation of extra-scientific reflection forms.
\end{abstract}

\section{Introduction}

Science, as a relatively independent social phenomenon, has, over more than 350 years of activity, gone through a complex and contradictory way from capturing society with its amazing results in discovering the world through anti-scientism after the bloody World War II with its mass destruction and the emergence of global problems that have been associated with scientific developments, to a more balanced assessment of its role in the life of society. For decades, philosophers, sociologists, cultural scientists, and representatives of other social and humanity sciences have been conducting scientific debate about the role of science in different social practices at different historical stages in the development of society and the science itself. There is no, and there cannot be unanimous assessment of this

\footnotetext{
* Corresponding author: drotlg@nau.edu.ua
} 
role, considering the differences both in the philosophical and worldview attitudes of the researchers, and in their recognition of certain socio-cultural factors as the preemptive ones in the social progress.

Systematization and classification of existing methodological, philosophical and worldview, sociological and other approaches to the reception of the role and functions of science in society, to determination of the influence of its basic structural components on the internal dynamics of science itself, as well as the place of socio-cultural factors in its development, helped to change the methodological understanding of the phenomenon of science in the last decades of the former century. In particular, V. Styopin's studies concerning the revolution in science itself, which led to changing the type of scientific rationality and at the turn of the XX and XXI centuries, contributed to the formation of post-non-classical rationality. The philosopher substantiated that "the foundations of science are, on the one hand, a component of the internal structure of science, but, on the other hand, of its infrastructure, which mediates the impact on the scientific cognition of socio-cultural factors and the integration of scientific knowledge in the culture of the corresponding historical era" [1]. The modern historical era is rightly called the information era, which corresponds to both the increasing role of computer science and information and communication technologies in the development of science itself, and the widespread use of these technologies in the social practices.

This actualizes the need to identify the relationship between the formation of post-nonclassical type of scientific rationality and science functions transformations in the context of informatization of society, which is the aim of this article.

\section{Materials and Methods}

As it is known from the philosophical and methodological literature, the historical periodization of science by types of scientific rationality was made by V. Styopin. Starting from the 1990s, he published several scientific works in which he argued that the development of modern science had taken place through four global revolutions: the first of them took place in the XVII century and marked the formation of classical natural science; the second, at the turn of the XVII and XVIII centuries, meant the formation of disciplinebased science; the third - from the end of the XIX to the middle of the XX century contributed to the formation of non-classical natural science; in the course of the fourth revolution - since the last third of the XX century - there was the formation of post-nonclassical science (highlighted by V. Styopin) [1]. According to these global scientific revolutions, in his opinion, such historical types of scientific rationality were formed in the history of science - classical, non-classical and post-non-classical [ibid]. According to V. Styopin, the post-non-classical type of scientific rationality broadens the reflection field over the activity. It takes into account the correlation of the obtained knowledge about the object not only with the peculiarity of the means and operations of the activity, but also with the value-target structures. With that, the connection of intra-scientific goals with extra-scientific, social values and goals is explicated. This type of scientific knowledge can be presented by the following scheme: 


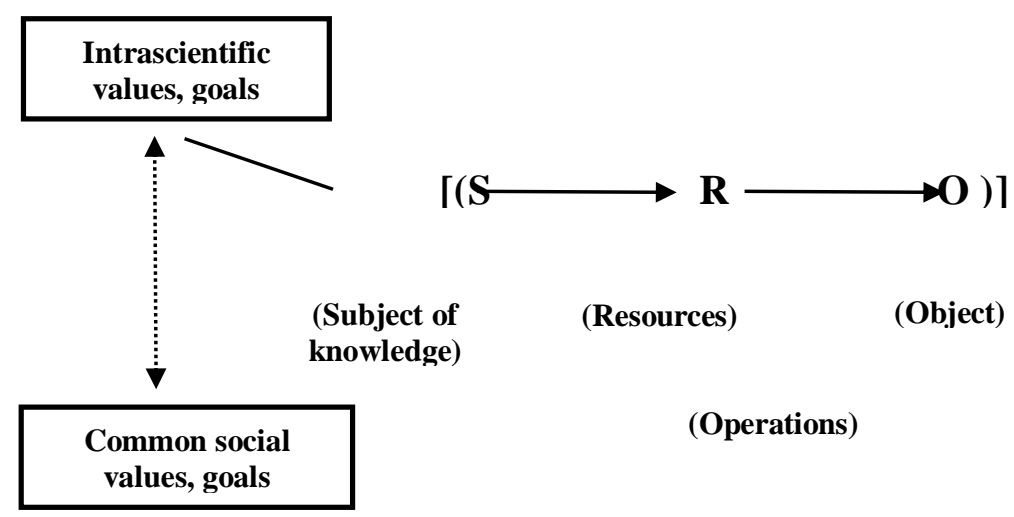

Fig. 1. Post-non-classical type of rationality (V. Styopin)

Based on this typology, national philosophers and science methodologists studied the features of its manifestation in various sciences. They were not limited by applying this methodology to the natural sciences only, but also applied it to understanding the development stages of the social sciences and the humanities. V. Arshinov, V. Budanov, I. Dobronravov, V. Kizima, O. Knyazeva, L. Mikeshina, B. Pruzhinin, V. Porus, M. Rozov, V. Rizhko, I. Tsekhmistro and others addressed this issue.

Understanding the specifics of the information society as a knowledge society is reflected in the works of D. Bell, M. Castels, J.-F. Liotar, V. Liakh, V. Onoprienko, E. Toffler, F. Webster, F. Fukuyama, S. Yagodzinskyi and many other foreign and domestic researchers. The peculiar result of these studies was the work of F. Webster Theories of Information Society [2]. He showed in it that not all the researchers of the information age society progress pointed to the role of science, theoretical knowledge as a defining feature of this era. However, in most studies of the basic characteristics of the information society, the transformation of the science functions, in particular, based on a post- non-classical type of scientific rationality, has hardly been analyzed.

\section{Results}

Neither scientists nor even ordinary citizens doubt that science played in the past and plays one of the leading roles in modern society. In fact, since the organic combination of science, technology and production as early as the XVIII century, the results of scientific knowledge have been widely used by all people at work, in everyday life, at leisure. However, deeper understanding of the science functions in the life of society began only at the end of the XIX century. Until then, the scientists, in particular F. Bacon and R. Descartes saw the aim of science in mastering nature and improving the human mind, using for this purpose, as it is known, different, even opposite, methods. At the same time, it is possible to find reasoning about the practical function of science in both of these scientists and philosophers. Thus, F. Bacon wrote that science has the task of implementing the prescriptions of practice [3]. R. Descartes noted the practical role of mathematics: "mathematics contains some very subtle devices that serve not only to satisfy those who are intrigued by mathematical problems but also to help with all practical and mechanical endeavours and to lessen men's labours" [4]. That is, at the time of the birth of classical natural science, scientists were already thinking not only about the cognitive function of science, but also about its social role.

If we summarize what functions were given to the science in its classical period, it should be noted that in the XVII-XVIII centuries the scientific knowledge fulfilled the worldview and educational functions. From the end of the XVI century, success in the 
newly-emerging classical science have contributed to the secularization of social consciousness. Religion was gradually supplanted by science, aimed at the earthly arrangement of man with the help of the science dependent technique. This plan of great renewal, put forward by F. Bacon, supported and developed by R. Descartes and others, became the basis of the project of the new time (modern). In fact, we can say that the belief in science, in its limitless possibilities in modern times, set the vector for the spread of atheistic and materialistic worldview, especially in the Enlightenment period.

Transitions from one historical stage of science to another required the revision of the methodological means of scientific knowledge. Methodology as an instrumental knowledge penetrates into the purposeful creative activity of the scientist, who constructs the researched reality on the basis of a reference, in the process of which he forms new scientific concepts, principles, approaches. Thus, if classical science, according to V. Kostyuk, was characterized by such concepts as "structure", "environment", "state", "interconnection", the modern post-non-classical science features has fundamentally new concepts - non-linearity, irreversibility, stability and instability, potentiality, critical behaviour, fluctuations and bifurcations, the whole, integrity and symmetry, selforganization, historicity, efficiency and Pareto-optimality [5]. Therefore, changing the conceptual characteristics of science significantly influences the change of its tools, including methodology.

Post-non-classical science in all the diversity of its branches, spheres, is based on the synergetic principles of research unified for this stage of scientific knowledge, because science now does not deal with closed, but rather with complex, open, non-equilibrium systems characterized by the phenomena which are characterized by the phenomena of instability, coherence, fluctuations, etc., the description and research of which are most adequately provided today by synergetic as an interdisciplinary science with its most common cognitive means based on quantitative methods. Another thing is that these methods are universal at a certain historical stage in the science development and the next stage is likely to develop new approaches and methods of knowledge. This is all the more important in view of strengthening the practical orientation of modern post-non-classical science. The very system of instrumental knowledge is becoming more open to new methodological tools, to the penetration into it of cognitive tools of other forms of knowledge (pre-scientific, para-scientific, religious, etc.).

As instrumental knowledge is immersed in subjective cognitive activity, it is connected not only with the researched objects, but also some of its elements are interconnected, they interpenetrate, complement and transfer into each other. This follows from the assumption that nature is not internally divided into separate sectors, and therefore its various parts should be explored by common cognitive means that complement each other. E. Laslo predicts the continuation of such trends in the XXI century. He writes: “... We can foresee the onset of the phase in the development of natural science when research, still limited by the frames of scientific disciplines, will be reinforced through the mathematical formulation of transdisciplinary dynamics, which drives evolutionary processes in various fields of observation" [6]. That means the formation in the future science of a unified system of research tools on the basis of the use of nonlinear mathematical equations, which are the basis for non-equilibrium thermodynamics studying the processes in the open systems of different origin.

It may look paradoxical at first sight, but it is mathematics and other deductive sciences and their cognitive tools that rely on the most abstract concepts, showing the best adaptation to the most extensive study of diverse nature phenomena. The problem is not the use of mathematics, but the question what kind of mathematics should be used, because it is commonly known that the mathematics evolution is so accelerated and has reached such abstraction heights that not all sciences are fast enough to catch up. 
It is appropriate to mention here that mathematics itself is an extremely complex system in which there are theories for the pure mathematics internal use only for the sake of its further development, but there are theories that become an effective means of developing other sciences. It is becoming increasingly difficult to distinguish these sections as computerization processes based on mathematical theories are invading wider and wider areas not only in science but also in practice; and mathematical theories previously considered "pure" are being transformed into such ones that find application not only in mathematics. That is, the processes that characterize the development of modern post-nonclassical science with its vague borders are becoming a defining feature both in mathematics and its applications.

For example, in the field of computational mathematics since the end of the XX century, when a high level of computerization and therefore, the use of numerical methods, is synonymous to high science in any field, the boundaries between its fundamental and applied sections are blurred. When solving any problem with the use of a computer, it is necessary to translate it from the language of algebra, geometry, mathematical analysis, topology, etc. into the language of numerical methods, choosing the most appropriate from the huge number of them. At the same time, it is not only important to find the right algorithm, but also to save the machine time as much as possible. So searching for the most effective tools is not only scientific but also purely economic problem.

In modern studies, mathematical tools can greatly influence the content and quality of the studied objects. Therefore, in post-non-classical science, very often mathematical theory in the process of its application for the search for new quantitative laws is also a method of scientific knowledge. More often this conclusion concerns the application of fundamental and applied instrumental knowledge in the process of computer simulation, where, for example, differential and integral calculus are intertwined with numerical theory, and fundamental mathematical theory can be transformed into an applied means of solving a particular problem.

True scientific progress is not a linear process, and therefore cognitive means cannot be placed in a certain invariable hierarchy, since they serve as the representations of scientific knowledge in reality. At various stages of the science development, often old tools were effectively used in new theories, and the new ones contributed to the further progress of the old theories. It is not accidental that in the conditions of post-non-classical science both scientists and methodologists keep saying not about a set of certain procedures, means, techniques of cognitive activity, but about the extension of these methods to methodological consciousness [7] which leads to changing the style of thinking, and in our time, in particular, before the emergence of the so-called nonlinear thinking, which also involves a moral evaluation of various methods of cognition.

On the other hand, a new methodological consciousness is beginning to produce new means of spiritual and practical development of reality that expand the possibilities of the human mind in creating a scientific picture of the world. H. Putnam calls N. Bohr's attempt to break the Kantian view of the Universe a striking manifestation of the methodological orientation change in the scientists' minds: According to Bohr, even the 'empirical world', the world of our experience cannot be fully described with just one picture. Instead, we should use 'additionally' different classic pictures, test them in different experimental situations, check partial pictures against the background of others, and come up with the idea of a unified concept that describes all situations [8]. The most modern, rather flexible, non-linear thinking, capable of forming a new scientific picture of the world, which would not claim to be comprehensive and would be able to sensitively respond to new discoveries and to yield to a new scientific vision not only of the outside world, but also of the human being place in it. This approach was taken by the creators of non-equilibrium 
thermodynamics and synergetics, which have seen in a new light not only evolutionary processes in the space after the Big Bang, but also developed an anthropic principle.

Although the trends of the scientific methods specialization in each sector remain in post-non-classical science, their integration seems to dominate over differentiation. In fact, the science of the future is likely to combine disciplinary and interdisciplinary research. Another thing is that their forward and reverse connections can be much more intense and their boundaries less rigid. In these situations, the overall scientific picture of the world will be increasingly understood as a global research agenda and a necessary horizon of the knowledge systematization. An important role in this research agenda belongs to further integration of special means of research, and in particular to general, interdisciplinary, fundamental and applied methods of quantitative analysis, as they can simultaneously describe phenomena of different nature.

In the science of information society, information and communication technologies, which are crucial not only in the natural and technical sciences, but also in the social sciences and the humanities, are becoming one of the common scientific cognitive means [9]. Moreover, computer technologies are also effectively applied in scientific study, which enables to identify the patterns of transformations that occur in the process of development of scientific knowledge. Information technologies together with cognitive sciences stimulated the development of new directions of epistemology research. The computer metaphor enables to explore the acquisition of knowledge, its preservation, processing, to identify the types of knowledge possessed by man and the methods of their use [10]. Their further development "changes the spatio-temporal characteristics of being, "materializes" virtuality as a form of consciousness being, ... habitual modes of activity and communication, stimulates the formation of new forms of organization, characterized by increased levels of complexity, the formation of sparse network structures that require special forms of management" [11]. Thus information and communication technologies acquire ontological status, becoming technological activities and at the same time representing objective reality.

In the classical period of the science development one of its important functions was educational function, because production sphere required educated engineering personnel, which was accompanied by the establishment of polytechnic schools and institutes, which invited scientists from various natural and mathematical branches of science to teach students. Since the Enlightenment epoch, science has always been legitimized through higher education, so it is not a coincidence that the greatest thinkers in various historical periods and in different countries have taken care of the problems of higher education.

The formation of non-classical science has further sharpened the attention of scientists to the educational function, because the problem of adapting the education system to the demands of industrial production was put on the agenda. In this context, the chain "science - technology - production" could only be continuous if it passes through the educational institutions, which were intended to provide such a level of education that would correspond to the level of science development. But the educational function of science at its non-classical stage have become important in the science itself. Especially in the twentieth century, scientific activity became collective, when scientific schools began to play a significant role in the development of scientific knowledge. The educational function of science in the activities of the latter was not to teach young scientists the methods of research, but to ensure the continuity in the scientific knowledge development from generation to generation by means of personal communication of famous scientists with scientific youth. In this way, cumulative-progressive process of scientific knowledge increase is carried out.

At the start of the twenty-first century, higher education in the United States stands as a formidable enterprise. As an established "knowledge industry" it represents about 3 percent 
of the gross national product" [12]. This enables us to determine what new knowledge is needed, whether it is appropriate to acquire such knowledge and what needs to be done to ensure its effective use. Moreover, only science is capable of accomplishing this mission, with the appropriate means in its arsenal. "The top three countries in terms of expenditure on fundamental science in relation to GDP are Switzerland ( $0.9 \%$ of GDP), South Korea $(0.76 \%)$ and Iceland $(0.65 \%)$. As for applied research, the picture is as follows: Currently, in the US, domestic R\&D spending is $2.8 \%$ of GDP, in China $-2.1 \%$, in Japan $-3.4 \%$, in South Korea $-3.8 \%$, in the most developed countries of Europe they are at the level of 2.5$4 \%$ " [13].

The main driving force of effective research are the scientific elites. The Challenge 2035 collected works authors [14] strongly suggest that the main determinant of the scientific elite cultivation is the sum of technologies used in practice in one's private and social life. Accordingly, all countries in the world are divided into three zones, depending on the dominant technologies. The first zone unites the countries in which the types of socially significant activity are based on the developed system of high technologies. It is the "green zone"; it creates the maximum conditions for the safe existence of scientists. The second type of society is the "yellow zone" in which measures are taken to minimize talent leakage and cultural self-isolation. The third one, the "red zone", is technologically and scientifically backward, so talented people, scientists, inventors, etc. tend to leave it. The metaphorical "color classification" of modern societies, proposed by the authors, is based on the analogy with the traffic lights system. "Green zone" means that the societies belonging to this zone have the prospect of a prosperous existence and rapid development of all sides of scientific, technological and cultural and civilizational social activity. The states that get into the "yellow zone", in order to move into the prosperous future, which provides conflict-free existence and the existence of conditions for self-realization of the individual, should make more or less significant efforts to accelerate scientific and technological progress and strengthen the social institutions that develop on its basis. As for the states of the "red zone", the same as in the traffic system, the red color of the social semaphore shows that the road to the future is closed.

The research on the scientific staff mobility shows that it is constantly growing. Particularly noticeable is the increase in mobility since 2000. The impact of international mobility on the economic characteristics of the scientific and educational systems has not yet been sufficiently studied, as it is a complex and multifaceted phenomenon. The studies conducted in the United States allow us to identify a number of economic effects, both positive and negative, arising from staff mobility. Table 1 from "Research and policy issues in high-skilled international migration: A perspective with data from the United States" [15] summarizes the main economic effects for the recipient countries (receiving foreign personnel) and donors (the countries which the personnel leave), as well as global effects that cannot be attributed to a single country.

Table 1. Possible global and national effects of high-skilled international migration

\begin{tabular}{l}
\hline SENDING COUNTRIES: POSSIBLE \\
NEGATIVES \\
•"Brain drain": lost productive capacity due to at \\
least temporary absence of higher skilled workers \\
and students \\
- Less support for public funds for higher \\
education
\end{tabular}
education
RECEIVING COUNTRIES: POSSIBLE NEGATIVES

- Decreased incentive of natives to seek higher skills

- May crowd out native students from best schools

- Language and cultural barriers between native and immigrant high-skilled workers

- Technology transfers to possibly hostile countries 


\section{SENDING COUNTRIES: POSSIBLE} POSITIVES

- Increased incentive for natives to seek higher

skills

- Possibility of exporting skills reduces risk/raises expected return of personal education investments

- May increase domestic economic return to skills

- Knowledge flows and collaboration

- Increased ties to foreign research institutions

- Export opportunities for technology

- Return of natives with foreign education and human capital

- Remittances and other support from diaspora networks

\section{POSSIBLE GLOBAL EFFECTS}

- Better international flow of knowledge

- Better job matches

- Greater employment options for workers/researchers

- Greater ability of employers to find rare/unique skill sets

- Formation of international research/technology clusters (Silicon Valley, CERN)

- International competition for scarce human capital may have net positive effect on incentives for individual human capital investments

In the process of transition of science from the non-classical to the post-non-classical stage, its social function is significantly enhanced. Researchers, in one way or another, have to touch not only upon the problem of the truth of the obtained knowledge, but also to evaluate its impact on the changing social conditions of the existence of each individual and society as a whole. In other words, the task is to identify the level of economic and sociocultural effectiveness of scientific knowledge. As J.-F. Liotard rightly points out, "we resort to a higher principle, a "metaprinciple," which bases the development of knowledge, of society and the state "in the realization of the "life' of a Subject" [16]. He writes that science becomes a productive force precisely in the period of its organic combination with capital, which provides investment in scientific development, which, in turn, guarantees profit. In any case, scientific research is of social value because it strongly influences both economic development and the spiritual existence of society.

A contemporary American philosopher of science M. Thompson, analyzing the transformation of the social role of science in modern society, argues that scientists are called to interpret scientific data, for example, to assess the safety of a food product or long-term use of a mobile phone impact on human health. After all, the public is convinced that science will give answers to all questions. It is important for people to know the professional opinion of what is or "acceptable risk" or "harmfull" [17], when they use the practical results of scientists' research.

The transformation of the social function of post-non-classical science forms new foundations for the activity of scientists in the scientific field itself. V. Porus's statement about new vectors of modern and future scientific research is valid. Really, he writes that the problems of intellectual and social responsibility, moral choice, personal aspects of decision-making, which have significant consequences for the whole social context in which the science and technology exist, problems of moral climate in "think tanks" (V. Porus) acquire the actual theoretical and cognitive sense [18]. Indeed, in modern scientific works, researchers are increasingly turning to the understanding of moral and social responsibility of scientists, especially in the natural and technical fields of science. There are the risks connected with the application of new mega-technologies: information, bio-, nano-, cognitive and socio-humanitarian ones. Thus, the knowledge society itself creates the risks that are associated with the incompleteness of the knowledge itself. That is, 
science and technologies created by it, on the one hand, are designed to improve human life, and on the other hand, contribute to the increase of uncertainty of the future. Therefore, one insists on the study of the anthropological aspect of the latest technologies. In this context, it can be argued that the transformation of the social function of post-non-classical science enhances its human-dimensional character.

In response to this challenge, policy-makers and scientific societies have continued to develop the necessary infrastructure, to issue and disseminate codes of conduct and guidelines for responsible research (RR), to develop educational programs, to hold conferences and congresses. The United States were the first to come this way. Following the US, similar structures and rules began to be created in European countries, especially in the United Kingdom, Denmark, France, the Netherlands, Germany, Finland, Portugal, and Sweden.

In the conditions of widespread informatization of society, one of the most important is the information function of science. So, P. Drucker notes that knowledge today is information that has practical value: [the functions of knowledge workers] "arise from the need to achieve effectiveness and competitiveness in organizations and in society as a whole. These functions are specialized, covering both the individual and social outlooks" [19].

New information technologies (IT) have attracted thousands of people around the world to participate in the process of acquiring new knowledge on a distributed, informal basis. Voluntary participation in knowledge creation is not a new phenomenon, however, it has received additional impetus with the appearance of modern IT due to the contribution of a large group of people, usually from online communities. Scientific crowdsourcing and the citizens science, which has grown out of crowdsourcing projects, is a research practice in which the public (non-professional scientists) cooperate with professional scientists in conducting scientific research. The earliest and the best-known platforms for supporting micro-tasks by distributed teams and individuals are Amazon Mechanical Turk, Stack Overflow and Freelancer.

More complex tasks are solved with the support of special crowdsourcing software, which enables projects to increase modularity; it becomes a condition of shaping the "citizens science". This is primarily Topcoder, Upwork and Crowdplat. As a rule, behind each type of such software there is a certain company that supports crowdsourcing platform for solving a certain task.

For cooperative testing of various parameters, participants download an application on their mobile phone that allows them to collect data. They use sensors that are already available in their mobile phones. These are various transceivers (mobile network, Wi-Fi, Bluetooth), FM and GPS receivers, cameras, accelerometer, digital compass and microphone. The examples of data provided by a "crowd" of agents include ecological quantitative data such as temperature and atmospheric conditions, the data from the analysis of an individual's daily behavior (for psychologists), interpersonal interaction data, such as crowd density or crowd elements interaction. This data also includes categorical recommendations, such as ratings of nearby restaurants, as well as users' comments on the wireless mobile multimedia applications.

The second most common direction in mass data collection is the refinement of various types of mapping data. Data collection from volunteer participant-observers in many projects overlaps with the data from mapping agencies, for example in OpenStreetMap application. There are also hybrid attempts to combine official mapping networks like LUCAS 1 with an array of volunteer contributions "armed" with mobile applications, such as FotoQuestAustria [20].

The analysis shows that not only IT technologies support crowdsourcing, but crowdsourcing also contributes to the improvement of IT infrastructure. Voluntary 
computing requires continuous improvement of parallel and distributed computation algorithms. Volunteering projects require the support of websites, forums, trainings, open databases and digital libraries.

The strengthening of information processes worldwide accompanies globalization that has swept across nations and continents. In this regard, scientists and philosophers believe that the development of information technology will in the future lead to the formation of the Collective Intelligence. In particular, this idea was expressed by M. Moiseev. He writes: "It is through the interaction of local intellects, the exchange of information between them, that the phenomenon of the Collective Intelligence and collective memory arose. Thanks to the possibilities of acquiring, accumulating and transferring knowledge it was possible for herd of 'smart' animals to transform into human society. [21]. He rightly believes that the current speed of information dissemination and exchange between people on different continents contributes to the development of shared assessments of events in the world, and ultimately leads to the "formation of a universal mentality" [Ibid] and the ability to make collective decisions. Therefore, we can conclude that this is not just about the information function of science for the purpose of disseminating knowledge, but about information as a socio-cultural phenomenon in the context of the development of scientific knowledge and its impact on all spheres of social life.

Apparently, this is what S. Krymskiy meant when he wrote that "the social mentality information-cooperative constituent of our time opens up operational and procedural possibilities of new thinking and enables it to be connected with both new types of sociocultural activity and the decision-making theory as an applied methodology for overcoming problem situations caused by the scientific and technological revolution" [22]. The philosopher warned that the possibilities of science and the introduction of its discoveries in social practices should not be uncontrolled by society, since it is necessary to take into account the smallest risks of practical penetration of scientific information in all cells of society.

Therefore, the purpose of modern post-non-classical science is not so much the cognitive value of knowledge about the human external world, but the priorities definition of socio-cultural nature. Such a change in the orientations of science leads to the fact that objectively true explanation and description regarding 'human-dimensional' objects not only allows, but also envisages the involvement of axiological factors in the explanatory provisions, the ethics of science, focusing on humanistic ideals and values of humanity is increasing [23]. Therefore, it can be said that post-non-classical science possesses a humanistic function, since "scientific achievements, especially built on the foundations of technology, become of humanitarian value, because they affect not only the solution of some particular problems, but also the ones that determine human existence, the meaning of its existence" [24].

The humanistic function of post-non-classical science is enhanced by the transformation of the methodological consciousness of scientists who are aware of their responsibility to society for the realization of scientific discoveries. "The concept of transhumanism, based on the latest achievements of biomedical technologies is one of these theories with ideological connotation successfully fitting into the context of new European ideas of progressive development. Treating a person as an intermediate product of biotechnological evolution, its supporters intend most of the current anthropological problems to be solved by a technological expansion of the mental and physical capabilities of a person and prolonging his life" [25]. By that the researcher meant precisely the fulfillment of the humanistic function of science by scientists.

However, one can't help noticing that the morally attractive idea of sharing knowledge of all countries and peoples of the planet contradicts the values of the main agent of global change - the technogenic civilization. It's cultural and genetic code is focused not on 
internal perfection, but on the active transformation of the world by technological means. Professor A. Sokolov insightfully observes that each side - humanistic and technocratic has its own "truth" and its own "untruth". Therefore, in his opinion, the information society turns out to be a social myth "about a global post-industrial intellectually developed society, which satisfies, through machine information technologies, the material and spiritual needs of the individual, social groups and the state" [26]. Being within this contradictory view, everyone can put the accents at his will.

The world is on the verge of a new technological revolution that, by its scale, will surpass all the wildest dreams and expectations. Converging nano-bio-informationcognitive (NBIC) technologies will play a leading role in the new technological landscape [27]. On their basis by the middle of the XXI century technologies will have taken possession of human nature, including the activities of consciousness. Artificial intelligence will reach the level of natural intelligence, and in some cases it will surpass it. Machinehuman hybrids - biologically-based cyborgs and humanoid-like androids that will come closer to cyborgs by their characteristics, will become more widespread. The further development of these technologies will copy the ICT development situation. As events unfold in this direction, the concept of the "information society", having played its historical role, is likely to go back into the past and give way to the concept of a "knowledge society" based on NBIC technologies, which will create virtually unlimited communication opportunities across the Earth.

The communicative function of science has always taken place in the history of its development and functioning in society. However, the humanistic context of this science function is making significant changes. M. McLuhan noted in his time that science had finally turned to the study of language as a means of communication that gives an orderly form to everyday life, so that society begins to look like a linguistic echo, or a repetition of language norms [28]. A specific Internet communication culture which is based on the observance of certain ethical norms of communication has emerged.

As one of the first researchers in the history of the Internet, M. Castells noted, technoelite formed the foundations of the Internet communication ethos. He states: "To be respected as a member of the community, and, even more so, as an authoritative person, technologists must abide by the formal and informal rules of the community and not use common resources (knowledge) or delegated resources (institutional positions) for their own exclusive benefit, beyond the shared good of advancing technological skills by learning from the network... Thus, the culture of the Internet is rooted in the scholarly tradition of the shared pursuit of science, of reputation by academic excellence, of peer review, and of openness in all research findings, with due credit to the authors of each discovery" [29]. It is obvious that the communicative function of science extends to the entire Internet society, not just to scientists, since all Web users are required to comply with ethical standards in the communication process, although communicators may not be personally acquainted.

It is commonly acknowledged among the researchers of the Internet nature and function that no new knowledge is born within the Network itself, but it allows any user to deepen his knowledge through communication. It is not just finding the information you need, including scientific information, but engaging in the dialogue with many users, expressing your own position, without fear of any harassment and negative evaluation. The only requirement in the process of such communication is compliance with the rules of communication culture. Scientists from different countries and continents in the process of virtual communication have the opportunity to test their scientific results on the basis of almost limitless audience of scientists and practitioners interested in the corresponding knowledge. 


\section{Discussion}

The realities of modern science do not allow to form its unambiguous image, reflecting the cognitive picture of the information society. On the one hand, we should acknowledge the interest in this issue and optimistic forecasts, both in relation to this research trend and the methodological arsenal for its promotion provided by the philosophy of cognition. On the other hand, there is concern about the processes that are deforming science and that lie in the socio-cultural and socio-economic aspects, where the changes in the value orientations of scientists, in turn, initiate serious the changes in the very essence of scientific activity and the phenomenon of science as a whole. The latter circumstance gives rise to a desire to contemplate on whether the information society is in fact the science and knowledge society, and what kind of science will be adequate to the offered socio-cultural realities.

\section{Conclusion}

The above study of the science functional transformations at its post-non-classical stage revealed several specific features.

First, the interdisciplinary nature of post-non-classical science has led to the transformation of cognitive function, which was reflected in the transition to the development of complex scientific programs, the implementation of which creates a special situation of merging in a single system of theoretical and experimental research, applied and fundamental knowledge, intensification of the direct links between them and feedback. This contributed to the creation of a comprehensive scientific worldview by common means of natural, social, humanitarian and technical sciences. In the conditions of the scientific cognition informatization process, computer technologies are added to the methodological means by which scientists have the opportunity to create virtual images of objects and phenomena whose analogues do not exist in objective reality, and thus to predict new facts and new objects. That is, with the help of their concepts relating to ideal entities the scientist constructs the real objects of his study setting a certain interpretation of external influence. Information and communication technologies make it possible to cover at one time large amount of information not only about the investigated object, but also about its connections and relations with other objects, which facilitates the discovery of new laws of the Universe.

Information and communication technologies also play a leading role in the science interdisciplinary branches formation that explore the most complex objects of different nature. Synergetics, non-equilibrium thermodynamics, informatics and cognitive sciences, which opened up new opportunities for technical and technological innovations in various social practices, have become the science frontiers at the turn of the II and III Millennia. In these fields of science, substantially new methodologies of scientific search have been formed, which are based on the transfer of cognitive models from one field of science to another with their subsequent correction according to the subject of research.

Second, the information function of post-non-classical science is transforming. It is connected not so much with the penetration of scientific knowledge in all spheres of public life (information), but with the need to constantly develop new scientific knowledge for the individual's self-realization through mastering new specialties based on the use of modern technologies, as well as conscious acceptance or, conversely, the rejection of new cultural phenomena that enter the society through modern information and communication systems.

Third, the information transformation function of post-non-classical science affects its fulfillment and educational role, since "education that will serve the information economy must be aimed at preparing the creative worker, with a developed imagination and innovative approach to the tasks set" [30]. 
Fourth, the science social function is undergoing significant changes. Society requires that scientists conduct professional scientific examination of various products for its safety for human health and the existence of the natural environment. The social function of science is also to reveal the risks posed to life by the development of scientific knowledge and modern high technologies generated by science.

Fifth (and the most important), all the post-non-classical science features intertwine and reinforce each other. In particular, the humanization of science contributes to its better fulfillment of social and communicative functions. At the same time, the communicative function increasingly acquires ethical colours, imposing bans on the use of scientific knowledge for selfish purposes. Thus, the information function of science is transformed, as the acquisition of scientific information becomes more accessible through information and communication technologies.

Thus, in post-non-classical scientific research, a number of significant changes are outlined, which include not only regulations related to non-classical ideals and norms of explanation and description, justification and evidence that take into account the relativity of an object to the means and operations of activity, but also those regulations that relate to overcoming disciplinary (subject) disunity. The boundary dividing particular branches of science becomes a uniting medium of communication, in which trans disciplinary and trans linguistic exchange processes are worked out, including value reflection and normative bases of scientific knowledge. In the new rationality, truth emerges as the practical basis of social consolidation, which always belongs to a particular nation, time, culture, etc., so, unlike the timeless truth of classical rationality, new rationality acquires very definite space and time.

\section{References}

1. V.S. Styopin, Philosophy of Science and Technology, 22(1), 7-11 (2017). doi: 10.21146/2413-9084-2017-22-1-5-29

2. Fr. Webster,. Theories of the Information Society. (London: Routledge, 2014) doi: doi.org/10.4324/9781315867854

3. Fr. Bacon, The Great Instauration And The Novum Organum (Literary Licensing LLC. 2014)

4. R. Descrates, Discourse on the Method (SMK Books, 2009)

5. K.N. Kostiuk, Professional education in the modern world, 6(4), 608-619 (2016). doi: https://doi.org/10.15372/PEMW20160406

6. A. Krushanov, Philosophical problems of information technology and cyberspace, 2(15), 111-127 (2018). doi: 10.17726/phillT.2018.2.15.7

7. T.P. Berseneva, Man and culture, 5, 35-46 (2013). doi: 10.7256/23061618.2013.5.9526

8. C. Jennifer, Revue internationale de philosophie, 218, 417-430 (2001).

9. L. Orochovska, , \& M. Abysova, MATEC Web of Conferences. 106,. 15-17 (2017). doi: 10.1051/matecconf/201710601005.

10. S.B. Krayniuk, Social psychology and society, 10(4), 23-41 (2019). doi: 10.17759/sps.2019100403

11. V.I. Onoprienko, Proceedings of the National Aviation University, 1(21), 27-30 (2015). doi: 10.18372/2412-2157.21.10664

12. J.R. Thelin, \& J. Edwards, Higher Education in the United States. Historical Development. System.

Retrieved

from 
http://education.stateuniversity.com/pages/2044/Higher-Education-in-UnitedStates.html.

13. I.G. Bokova, UNESCO. Director-General, 2009-2017 (2016).

14. I. Agarmirzian, D. Belousov, E. Kuznetsov, (et al.). Call 2035. (V.V. Burov (Ed.). Moscow: Olimp -Business, 2016).

15. M.C. Regets, Research and policy issues in high-skilled international migration: A perspective with data from the United States (Institute for the study of labor, Bonn, 2001).

16. B. Gaëlle, Cités, 45, 87-101 (2011). doi: 10.3917/cite.045.0087

17. Th. Mel, Philosophy of Science: Teach Yourself (John Murray Press, 2012).

18. V. Porus, Epistemology \& Philosophy of Science, 55(2), 75-93 (2018). doi: 10.5840/eps201855230.

19. A. Turriago-Hoyos, Ulf Thoene, A. Surendra, SAGE Open, 6(1), 1-9 (2016). doi: $10.1177 / 2158244016639631$.

20. J.C. Laso Bayas, Remote Sensing, 11(8), 905 (2016)

21. L. Drotianko, Proceedings of the National Aviation University, 2(24), 14-18 (2016). doi: 10.18372/2412-2157.24.12536

22. L. Drotianko, Bulletin of the National Aviation University, 2(22), 10-13 (2015). doi: 10.18372/2412-2157.22.12312

23. A. Wrigley, J. Hughes, M. Sheehan, (et al.). European Textbook on Ethics in Research. Luxembourg (Publications Office of the European Union, 2010).

24. I.P. Skyba, Proceedings of the National Aviation University, 2(16), 63-66 (2012). doi: $10.18372 / 2412-2157.16 .9470$

25. E.A. Sergodeeva, Philosophical Sciences, 11, 55-69 (2018). doi: 10.30727/0235-11882018-11-55-69

26. M.N. Kolesnikova, Library science, $2, \quad 123-125 \quad$ (2013). doi: https://doi.org/10.25281/0869-608X-2013-0-2-123-125

27. I.V. Danilin, RUDN University Bulletin, 17(3), 555-567 (2017). doi: 10.22363/23130660-2017-17-3-555-567

28. M. McLuhan, Understanding Media: Human External Extensions (Gingko Pr Inc; Critical edizione, 2008).

29. M. Castells, The Internet galaxy. Reflections on the Internet, Business and Society (NY: Oxford University Press, 2003).

30. A.D Ursul, T.A. Ursul, Evolutionary paradigms and models of education of the XXI century (Modern Education, 2012). 